\title{
Spontaneous Resolution of Prenatally Diagnosed Pleural Effusion: A Case Report
}

\author{
Slagjana Simeonova Krstevska ${ }^{1}$, Dimitar Getov ${ }^{2}$, Petranka Nikova ${ }^{3}$, Igor Samardziski ${ }^{1}$, Aneta Sima ${ }^{1}$, \\ Irena Todorovska ${ }^{1}$, Vesna Livrinova ${ }^{1}$, Vlatko Girevski ${ }^{1}$ and Daniel Milkovski ${ }^{1}$ \\ ${ }^{1}$ University clinic for obstetrics and gynecology, Skopje, N. Macedonia
}

${ }^{2} \mathrm{PHO}$ "Dr Getov" Valandovo, N. Macedonia

${ }^{3}$ General hospital Gevgelija, N. Macedonia

\begin{abstract}
Pleural effusion is a general term for the accumulation of fluid in the pleural space. Incidence is approximately $1 / 15000$ pregnancies. It is frequently associated with extra thoracic anomalies, abnormal karyotype and congenital heart disease. The mortality rate is $53 \%$ but in cases with associated hydrops may be as high as $95 \%$. Spontaneous resolution or regression has been reported in 9 to $22 \%$ and has been associated with nearly $100 \%$ of survival. Intrauterine interventions such as thoracocentesis and pleuroamniotic shunting are considered to avoid progression of an otherwise potentially fatal disease. The presence of hydrothorax does not influence the mode of delivery and cesarean section should be reserved for obstetrical indications. We present a case of 36-year-old woman (G2, P1) referred to University obstetrics and gynecology clinic due to bilateral fetal pleural effusion in 28 gestational weeks. Standard ultrasound biometric parameters were adequate, Doppler of the fetomaternal unit, TORCH and amniocentesis for fetal karyotype were normal. Serial ultrasounds follow up was performed and there was a total reduction of the pleural fluid in 34gw. She was delivered in 38gw by cesarean section due to polyhydramnios, pedalic fetal lie and macrosomia. Neonatal outcome was uneventful and there were no signs of neonatal respiratory distress. Fetal pleural effusion is a rare but potentially fatal condition. Spontaneous resolution is possible and most desirable occasion.
\end{abstract}

KEYWORDS: Fetal pleural effusion; Spontaneous resolution

\section{INTRODUCTION}

We present a case of a 36-year-old woman (G2, P1) refered to university obstetrics and gynecology clinic for further assessment of bilateral fetal pleural effusion in 28 week of gestation.

From the medical record she had a history of laparoscopic resection of ovarian endometriosis at the age of 20 . At the age of 25 she had electrophysiological study and ablation due to heart rhythm disturbance. Afterwards she was regularly checked at cariologist with regular heart rhythm. Previous pregnancy was uneventful and resulted in spontaneous term birth of a healthy newborn.

In the actual pregnancy she received standard prenatal care according to the national guidelines. In our evaluation maternal investigations included full blood count, blood group and antibody screen. Morphology ultrasound assessment showed a single fetus with adequate growth for gestational age, normal fetal movement and echogenicity of the placenta.

Bilateral fetal pleural effusion was confirmed with an echogenic fluid collection in the fetal chest and diaphragmatic contour with maximal diameter of $10 \mathrm{~mm}$ (Figure 1-3).

Fetal echocardiography was performed by a certified maternal fetal medicine specialist. Associated cardiac and extracardiac anomalies were excluded. The presence of fetal anaemia was excluded by assessment of middle cerebral artery Doppler peak systolic velocity. TORCH was negative for infections (Toxoplasmosis,
Quick Response Code:

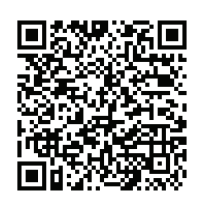

Address for correspondence: Slagjana Simeonova Krstevska, University clinic for obstetrics and gynecology, Skopje, N. Macedonia

Received: October 11, $2021 \quad$ Published: October 29, 2021

How to cite this article: Slagjana SK, Dimitar G, Petranka N, Igor S, Aneta S, Irena T, Vesna L, Vlatko G and Daniel M. Spontaneous Resolution of Prenatally Diagnosed Pleural Effusion: A Case Report. 2021- 3(5) OAJBS.ID.000343. DOI: 10.38125/OAJBS.000343 
rubella, cytomegalovirus, herpes simplex serology). Amniocentesis for fetal karyotype was negative for numeric aberrations of 13,18 and 21 chromosomes. Oral glucose tolerance test $75 \mathrm{~g}$ was negative for gestational diabetes.

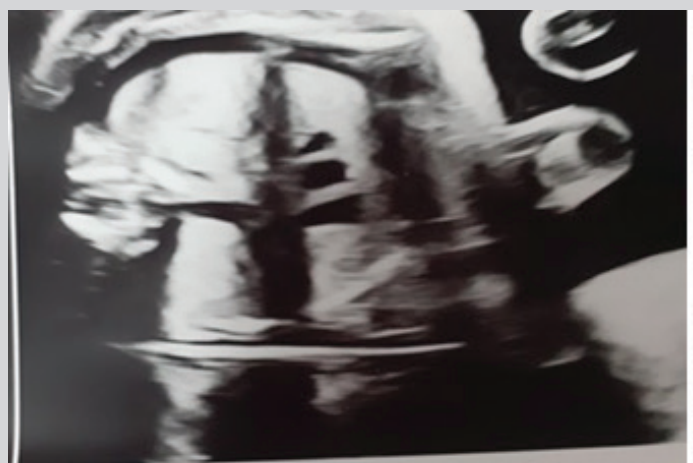

(1)

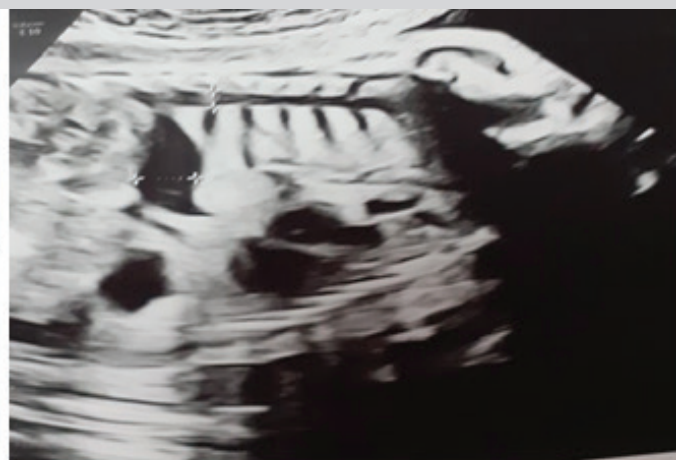

(2)

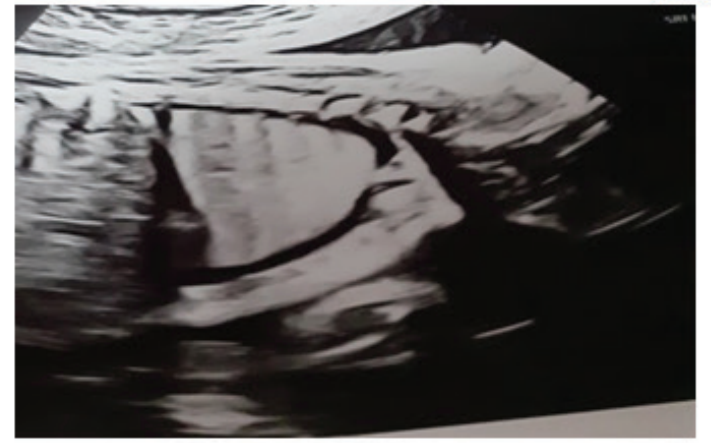

(3)

Figure 1-3: Ultrasound images of the pleural effusion.

Controlultrasound in 2 weeks interval showed polyhydramnious with an amniotic band (Figure 4).

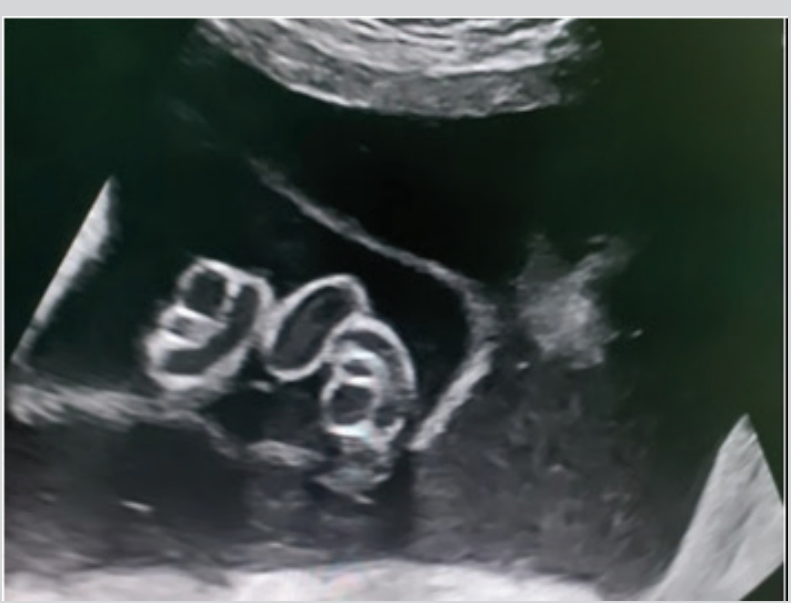

Figure 4: Polyhydramnious with amniotic band.

There was a total reduction of the pleural effusion in 34 gestational weeks with a complete resolution of the fluid (Figure $5,6)$.

In 38 gestational week she was delivered by cesarean section due to pedalic fetal lie, polyhydramnious and macrosomia. Newborn with fetal weight of 4030 , length of $52 \mathrm{~cm}$ was delivered with Apgar score 8/9.

Operative and postoperative period was uneventful. The newborn was discharged in good condition with normal respirations and no signs of respiratory distress.

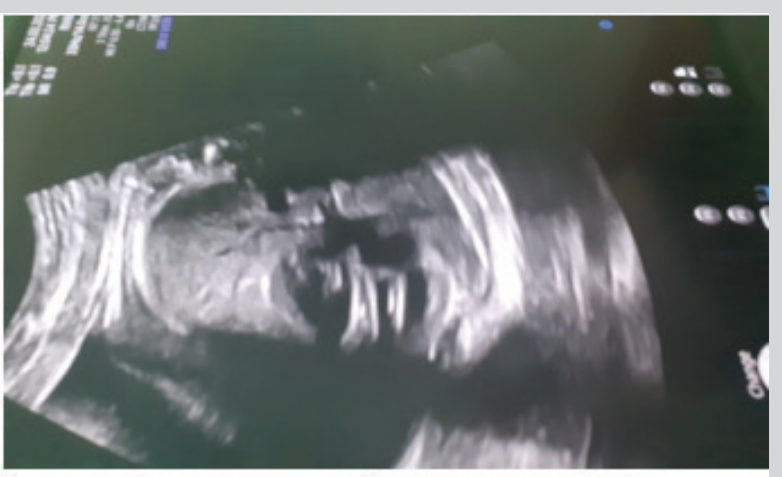

(5)

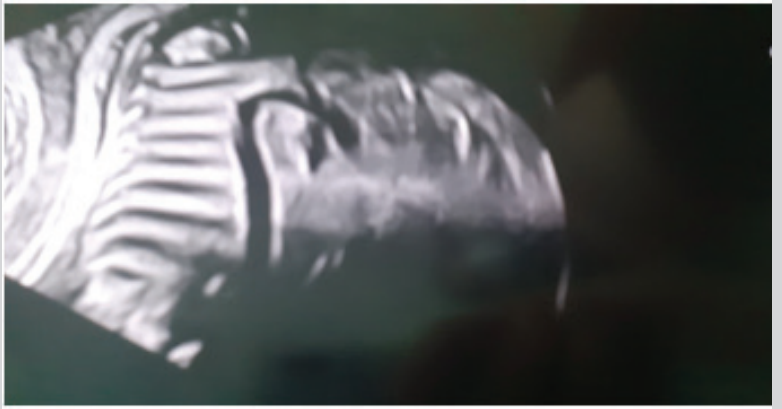

(6)

Figure 5,6: Complete reduction of pleural fluid in $34 \mathrm{gw}$.

\section{DISCUSSION}

Congenital hydrothorax is an accumulation of fluid in the 
pleural cavity. It is a rare disorder (1:10 000-15 000 births) with various causes (Noonan's syndrome, chromosomal anomalies, immunological, heart failure, extrathoracic anomalies, idiopathic). Fetal hydrothorax may be primary (chylous leak) or secondary (part of a generalized fluid retention in association with immune or non-immune hydrops). The risk of an abnormal karyotype up to $7 \%$ and risk for Down's syndrome around $4.9 \%$. On the other hand, the incidence of associated congenital heart disease may be as high as $5 \%[1]$.

Spontaneous resolution has been reported in 9 to $22 \%$ has a survival rate of nearly $100 \%$ [2]. In general, isolated fetal pleural effusions have a poor outcome, with neonatal death rates varying from $55 \%$ when diagnosis is made before $32 \mathrm{gw}$ to $30 \%$ when the diagnosis is made later. Mortality may rise close to $100 \%$ when hydrops is associated.

To predict which effusions will resolve or progress a period of observation and follow-up is warranted. Of all the poor prognostic indicators, hydrops is most important. It develops from mediastinal shift, cardiac compression and vena caval obstruction which diminishes venous return to the heart resulting in low-cardiac output state.

Even in the absence of hydrops, large pleural effusions can cause pulmonary hypoplasia due to compression and that is the most common cause of neonatal death.

\section{MANAGEMENT OF PREGNANCY}

Prenatal karyotyping is recommended, and a meticulous fetal echocardiography is needed [3]

A follow- up is advised with ultrasound examination in 2 weeks interval for detection of tension hydrothorax (mediastinal shift, diaphragmatic eversion), hydrops, and hydramnios.

It seems convenient that fetuses with large pleural effusion should be delivered in a tertiary-care center. Prenatal consultant with a pediatric surgeon, geneticist, and pediatric cardiologist may be indicated. The presence of hydrothorax does not influence the mode of delivery so cesarean delivery should be reserved for obstetrical indications.

\section{FETAL INTERVENTIONS}

The goals of antenatal therapy are: prevention of lung compression, prevention or reversal of hydropic changes and hydramnios, avoiding fetal death and preterm delivery, improved postnatal respiratory function.

\section{Thoracocentesis}

It is a diagnostic maneuver to obtain pleural fluid for cell count, differential, and culture and establish whether the effusion is chylous. It was proposed for the first time by Peters et al. in 1982. Clinical outcomes after fetal thoracocentesis alone are variable. Aubard et al. reported that $55 \%$ of fetuses treated by thoracocentesis had good outcomes [4]. Others have had disappointing results because of rapid accumulation of the effusion and neonatal death from respiratory insufficiency. However, if the effusion reaccumulates, thoracoamniotic shunting is felt to be less traumatic than repeated aspiration.

\section{Pleuroamniotic Shunting}

First proposed by Seeds and Bowes in 1986 should be considered for fetus $<32 \mathrm{gw}$ by creating a communication between the pleural space and the amniotic cavity [4]. This allows the lungs to expand, potentially reducing the risks of pulmonary hypoplasia, reducing pressure on the venous system, increasing venous return to the heart and improving coexisting heart failure.

A double pigtail catheter is passed through the trocar near the midaxillary line of the fetus, the "internal" loop placed in the thorax and the "external" end in the amniotic space. Most series reported survival rate 50 and $75 \%$.

Complications for thoracocentesis and thoracoamniotic shunts are fetal hemorrhage, abruption, migration of the catheter (intraamniotic cavity, maternal peritoneal cavity, neonatal subcutaneous tissue, neonatal intrathoracic cavity), infection, premature rupture of membranes, preterm labour. Procedure risks are increased by maternal obesity, hydramnios or unfavorable fetal position.

\section{Ex utero intrapartum treatment (EXIT)}

Allows thoracocentesis on the neonate while maintaining fetoplacental circulation. It may be useful for severe pleural effusion in late gestation when in utero drainage is not possible and drainage post-partum would result in profound hypoxia [5].

\section{Treatment with 0K-432 (Picibanil)}

Is a drainage of the pleural effusion and instillation of 0.1-0.3 KE/kg EFW of OK-432 (Chugai Pharmaceutical Co., Tokyo, Japan) in the pleural space? The procedure is repeated if there is no resolution of the effusion after 2 weeks. OK-432 is promoting a localised inflammatory response which cause pleural inflammation, fibrosis resulting in pleurodesis [6]. This is an experimental treatment as its safety remains to be clarified. However, it can be a valid treatment option for fetal pleural effusion with or without hydrops.

\section{CONCLUSION}

The clinical course of this condition is highly variable, ranging from complete resolution with good outcome to progression to hydrops fetalis and perinatal death. This condition is rare in prenatal medicine, but it does nevertheless warrant special attention. Our case is an example of a spontaneous regression and favorable outcome of fetal pleural effusion.

\section{REFERENCES}

1. Vera T, Coutinho-Borges JP, Carlos-Alves M, Santos J, Pinheiroa P (2017) Fetal primary hydrothorax with spontaneous resolution. Case Rep Womens Health. 15: 6-7.

2. Kajdy A, Materny A, Modzelewski J, Baszczeska J, Rabijewski M, et al. (2019) Spontaneous resolution of prenatally diagnosed isolated pleural effusion: An unusual early sign of a newborn disease, case report. The Journal of Obstetrics and Gynaecology Research. 45(12): 2466-2469.

3. Weber AM, Philipson EH, Ingardia CJ (2009) Spontaneous resolution of second trimester fetal pleural effusion. J Maternal-Fetal Neonat Med 1(2): 87-89.

4. Castagno R, Carreras E, Toran N, Higueras T, Sánchez MA, et al. (2007) Fetal pleural effusion. Donald School J Ultrasound in Obstet Gynecol 1(1): 28-39.

5. Prontera W, Jaeggi ET, Pfizenmaier M, Tassaux D, Pfister RE (2002) Ex utero intrapartum treatment (EXIT) of severe fetal hydrothorax. Archives of Disease in Childhood - Fetal and Neonatal Edition 86(1): 5860.

6. O”Brien B, Kesby G, Ogle R, Rieger I, Hyett JA (2015) Treatment of primary fetal hydrothorax with OK-432 (Picibanil): Outcome in 14 fetuses and a review of the literature. Fetal Diagn Ther 37(4): 259-266. 